\title{
Numerical Simulation and Analysis of the Arrangement of Wind Direction Meter for Wind Turbine
}

\author{
Zhipeng Xue ${ }^{1, *}$, Jie $F u^{2}$, Minmin Zhao ${ }^{3}$ \\ ${ }^{1}$ PH.D, Huadian Electric Power Research Institute co. LTD, NO. 2 Xiyuan 9 Road, Xihu District, Hangzhou Zhejiang Province, 310030 , \\ China \\ ${ }^{2}$ PH.D, Nanjing Gongdao Software Technology co. LTD, NO.17 YuJinxiang road, yuhuatai district, Nanjing, 210000, China \\ ${ }^{3}$ Engineer, Huadian Electric Power Research Institute co. LTD, NO. 2 Xiyuan 9 Road, Xihu District, Hangzhou Zhejiang Province, 310030 , \\ China
}

\begin{abstract}
This paper performs CFD numerical analysis on the wind direction variation characteristics of the upper part of the platform under the running condition of the whole machine, and analyses whether the current location of the equipment is suitable for placing the wind direction measuring equipment and finding a better position in the area. The vicinity of the reference point is the area where the airflow is relatively intense, and the wind direction is complex and changeable. In-depth CFD research on this flow phenomenon can obtain an intuitive wind direction state and inherently subtle changes.
\end{abstract}

\section{Introduction}

The wind direction meter is a high-precision measuring instrument for measuring the wind direction of the wind turbine in the wind field and is used for measuring the deviation angle between the wind direction and the central axis of the impeller[1]. The controller adjusts the rotation axis of the fan impeller according to the wind direction deviation signal through the yaw system to match the wind direction. The purpose of this paper is to maximize the efficiency of wind energy utilization, reduce the impact of wind direction changes on wind turbine load, achieve better power output, and improve unit efficiency. [2-5].

The wind direction meter is mounted on the top weather frame of the nacelle cover, which is susceptible to interference from blade rotation. Therefore, the actual power curve of the unit is seriously affected, so that the output power of the unit cannot meet the design requirements, and the utilization of wind energy and the efficiency of the unit are reduced[6-8]. The purpose of this paper is to use numerical simulation to select the best wind direction arrangement position on the nacelle cover to minimize the interference of the blade rotation on the wind direction meter. Ensure the safe and economic operation of the unit[9-12].

\section{Model description}

First, based on the analysis of a working condition, the effectiveness of the calculation process and method is investigated. Make an alternative layout point for analysis and comparison.

According to the provided three-dimensional digital model, the mesh model required for CFD calculation is established.

This project uses the relevant geometric functions of CATIA and Hypermesh to complete the geometric cleaning of the original digital modules of the main components blades, hubs, towers and nacelles, and assembles the components into a complete machine model. Based on the previous geometric cleaning, the Hypermesh related surface mesh creation function is used to divide the surface mesh. We used the trimming grid function in STAR-CCM +. The volume mesh required for the calculation is generated for the outer flow field area and the blade rotation area of the wind turbine, and the boundary layer of the wind turbine surface is simulated using the grid in the form of a prism.

\subsection{Establishment of flow field model}

The outer flow field is established by the boundary of the flow wall with the blade, the hub, the nacelle and the tower surface. The blades and hub are rotating mechanisms throughout the analysis, creating a rotating grid in this area. The $3 \mathrm{D}$ geometric model of the whole machine is shown in Fig 1 .

\footnotetext{
$\overline{{ }^{*} \text { Corresponding author: 34080575@qq.com }}$
} 


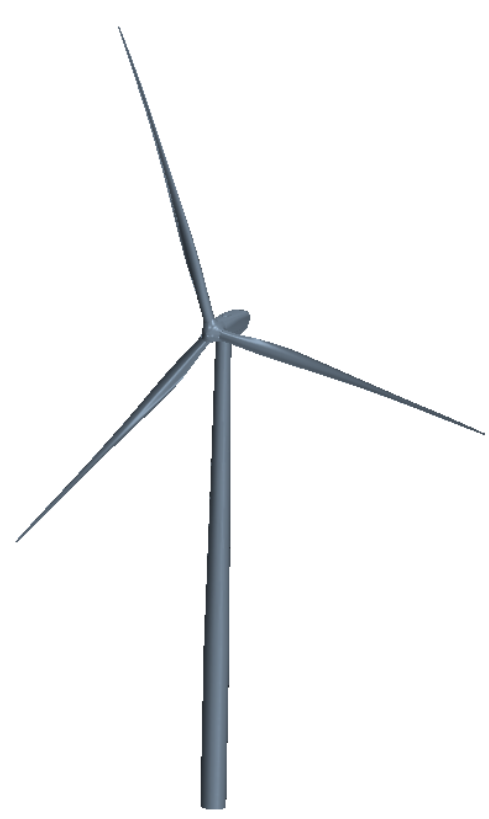

Fig. 1. Wind turbine model

The calculation domain of the whole machine is divided into two parts, the first part is the rotating area of the wind wheel, and the second part is the flow area of the external flow field. The enclosed area of the whole machine and the blade wind wheel is shown in the figure below.
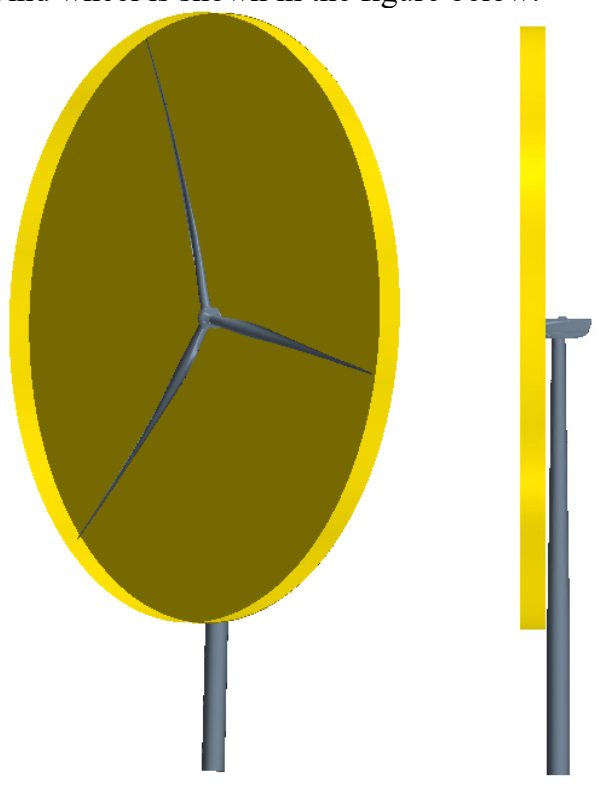

Fig. 2. Blade and wind wheel area model.

The wind tunnel model is shown in Fig 3. The wind tunnel size is 1100 meters (length) $* 600$ meters (width) $* 400$ meters (height).

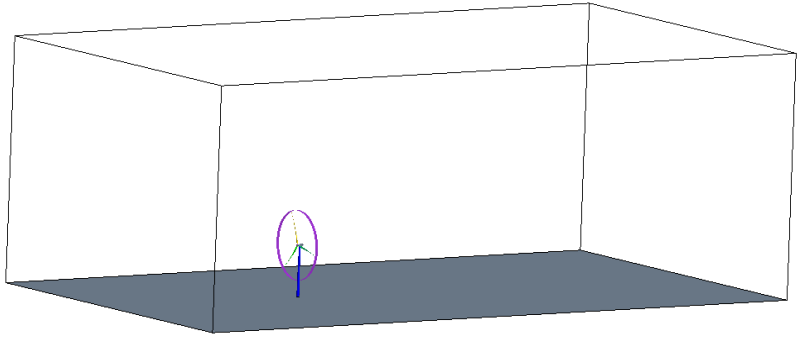

Fig. 3. Wind tunnel model.

\subsection{Face meshing}

The surface mesh is divided by the triangle mesh type. The division requirements are: no free edges, no repeating elements, the minimum angle of the mesh is greater than $20^{\circ}$; the specific settings of the mesh size are shown in the following table.

Table 1. Surface mesh size of each part of the whole machine.

\begin{tabular}{|l|c|}
\hline Part Name & Face Grid Size (mm) \\
\hline Cabin, hub & 100 \\
\hline Blade & $50-100$ \\
\hline Tower & 200 \\
\hline Wind tunnel 1 wall & 10000 \\
\hline Wind tunnel 2 wall & 4000 \\
\hline
\end{tabular}

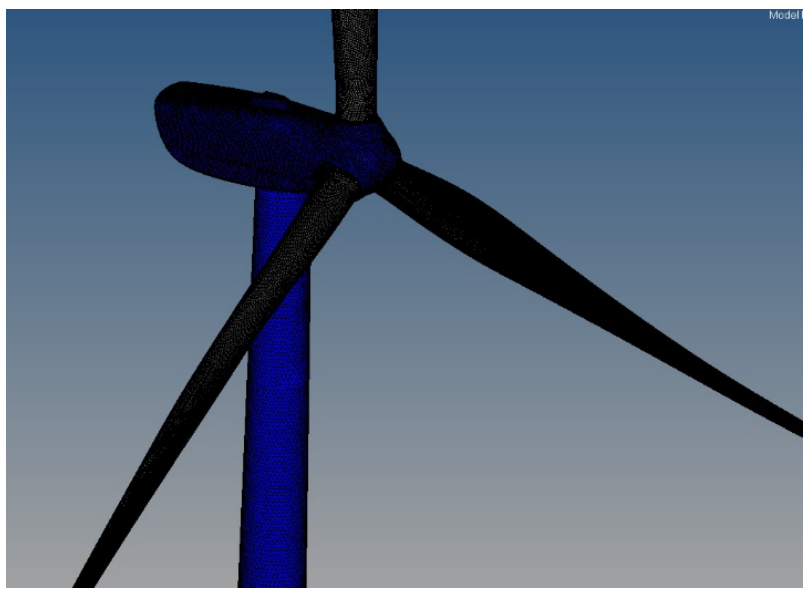

Fig. 4. Whole machine surface grid. 


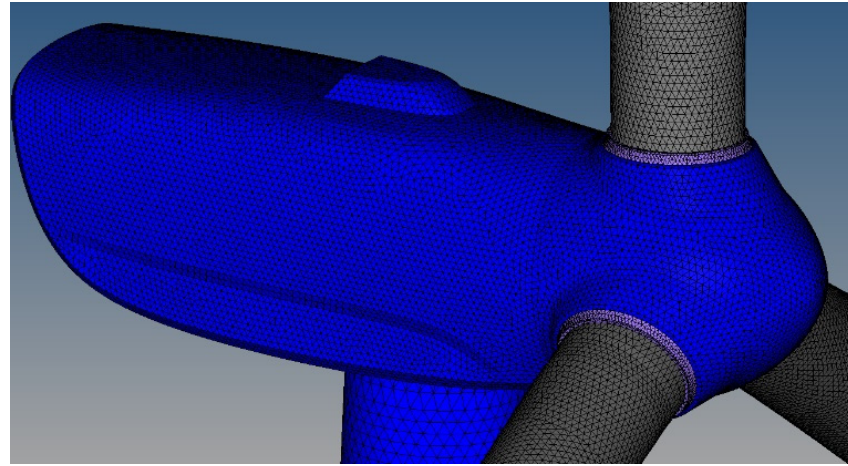

Fig. 5. Grid of the cabin hub.

\subsection{Body grid}

The volume mesh adopts the unstructured hybrid grid layout. The specific parameters of the boundary layer and multiregion encryption are: when the total thickness of the boundary layer is $1 \mathrm{~mm}, 2$ layers, the growth ratio is 1.3 。 The size of the encryption area is 100-200 near the cabin, and the tail The flow area is $0.6-1 \mathrm{~m}$. After repeated debugging, more than 9.4 million body meshes are finally generated, of which the blade rotation area is 3.4 million.

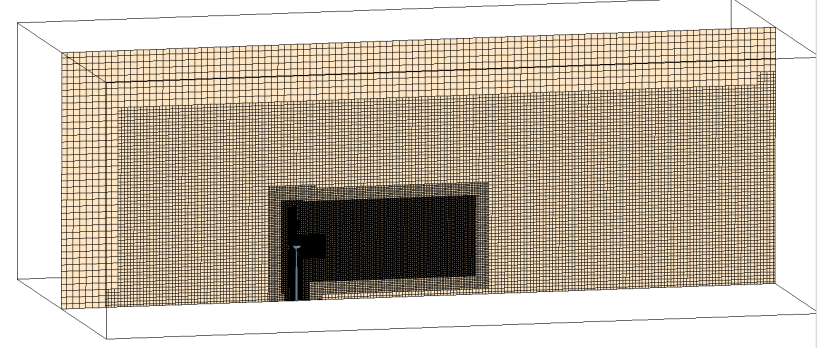

Fig. 6. Cross-section of the side body of the fan.

\section{Calculate parameters and boundary conditions}

\subsection{Environmental parameters}

The environmental conditions for the simulation calculation are shown in the following table and Fig 7.

Table 2. Environmental conditions

\begin{tabular}{|l|l|}
\hline The wind speed at the hub & $8.96 \mathrm{~m} / \mathrm{s}$ \\
\hline The height of the hub & 89.8 meters \\
\hline Wind shear parameter & 0.2 \\
\hline Wind direction & $8^{\circ},-8^{\circ}$ \\
\hline Fan speed & $15.2 \mathrm{rpm}$ \\
\hline
\end{tabular}

There is no need for vents and vents in the computer compartment. The flow rate at the vent is 10,800 cubic meters per hour.

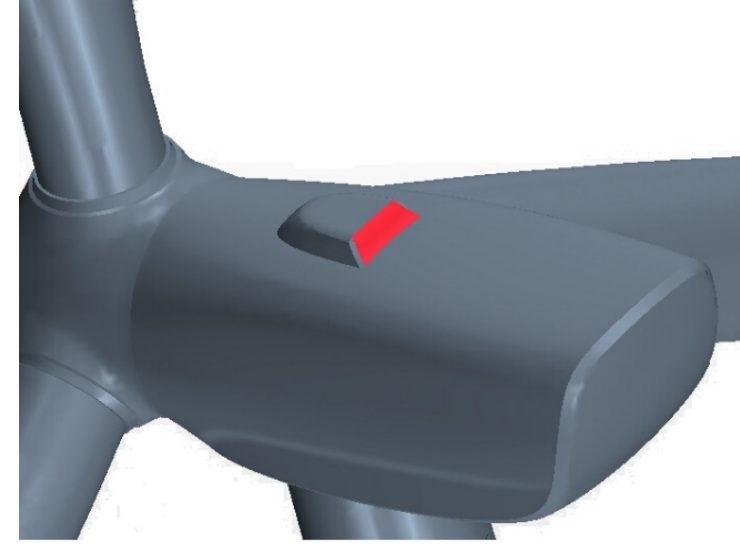

Fig. 7. Vent position (red area).

\subsection{Calculation parameters}

1. Calculate the gas flow in the domain according to the incompressible, constant flow calculation, using a doublelayered wall function,

2. The inlet is the speed import boundary condition, and the wind shear is considered when the inlet speed of the hub is $8.96 \mathrm{~m} / \mathrm{s}$. Free flow boundaries are used around the exit and flow fields.

3 . The convergence accuracy of the calculation is 0.001 , and the second-order upwind difference format is adopted.

4. Transient calculation: The time step is $0.025 \mathrm{~s}$, the maximum number of iteration steps in the unit step is 20 , and the total physical time is 60 seconds.

\section{Analysis of calculation results}

\subsection{Transient results}

The Fig 8 shows the real-time streamline diagram of the whole machine. It can be seen from the figure that the blade is the main source of interference in the transient, followed by the platform. The position near the reference point is the position where the airflow is relatively sharp, and the indepth CFD study is very necessary. 


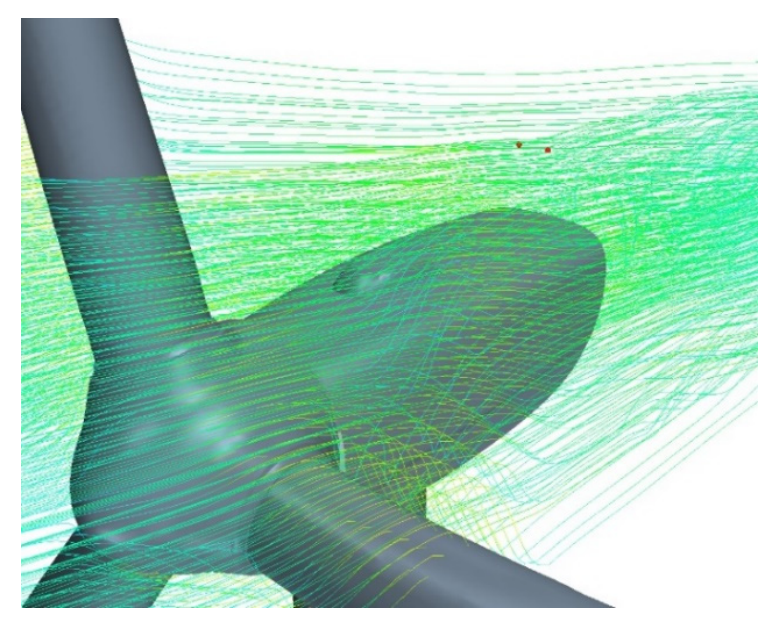

Fig. 8. Real-time streamline.

According to the reference point data of the wind vane position, at the upper equipment of the platform, two observation points are established, namely, L ref, $\mathrm{R}$ ref in Fig. 9, and the data to be optimized is output (pink area).

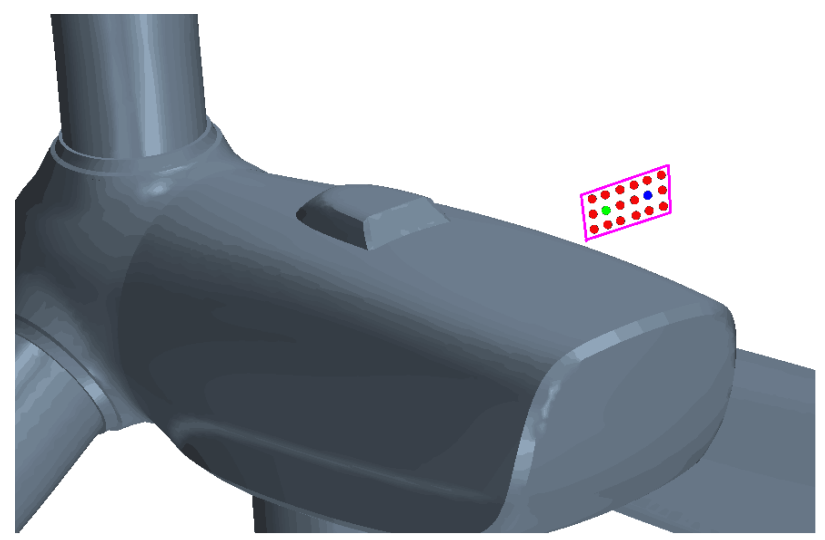

Fig. 9. Data monitoring points and areas (blue point is L ref, green point is $\mathrm{R}$ ref).

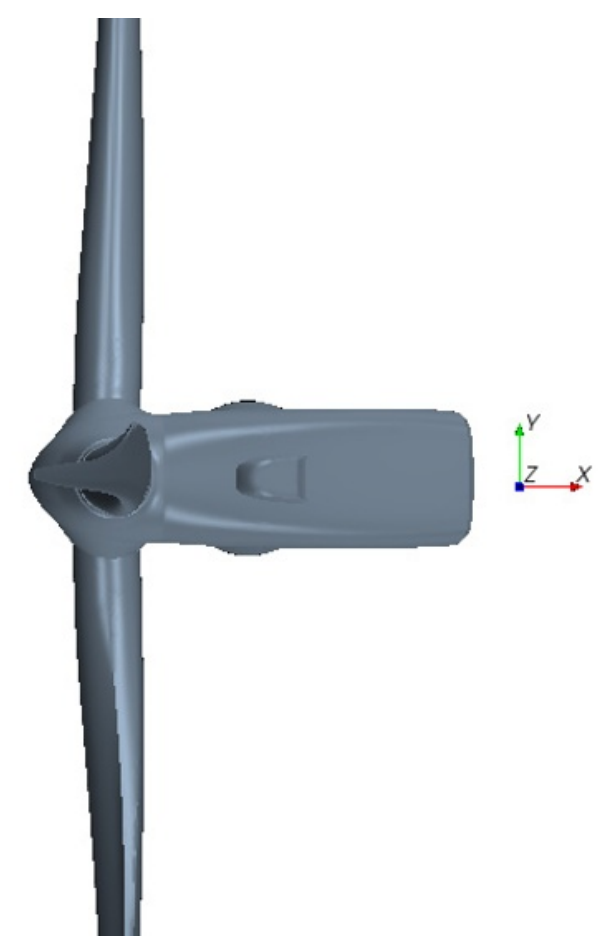

Fig.10. Model coordinate system definition.

Fig 10 shows the definition of the model coordinate system. After the calculation is completed, the wind speed detection data of the observation points $\mathrm{L}$ ref, $\mathrm{R}$ ref is derived, and the wind direction instantaneous angle is calculated. The wind direction instantaneous change data is created as shown in the following Fig11-14.

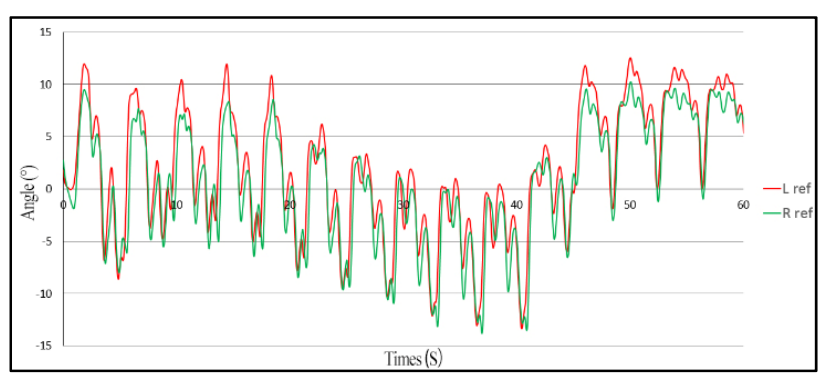

Fig.11. No vent $8^{\circ} \mathrm{L}$ ref, R ref real-time wind direction comparison chart.

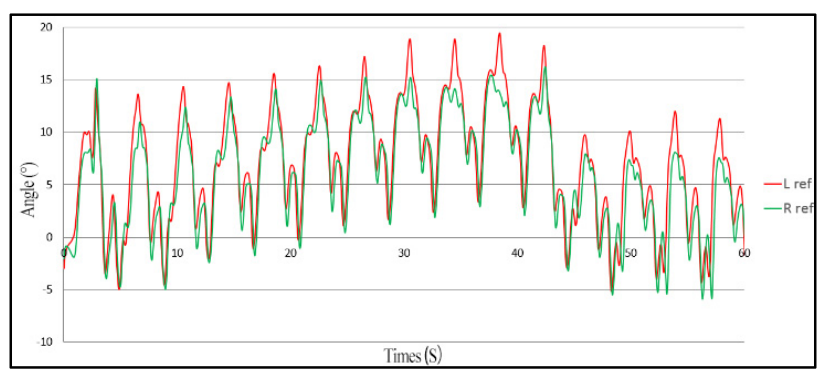

Fig.12. No vent $-8{ }^{\circ} \mathrm{L}$ ref, $\mathrm{R}$ ref real-time wind direction comparison chart. 


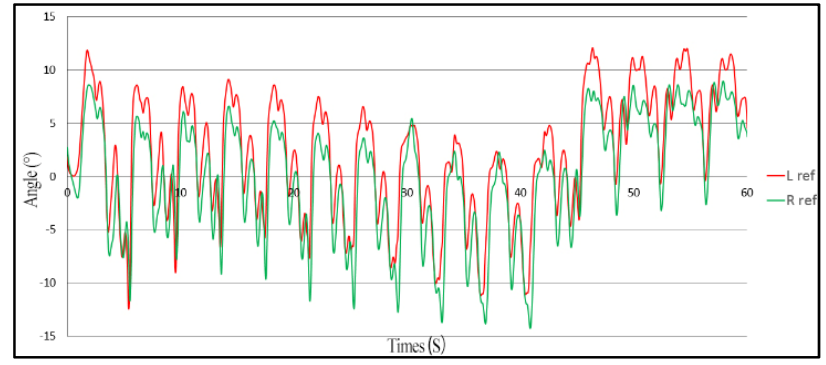

Fig. 13. vent $8^{\circ} \mathrm{L}$ ref, $\mathrm{R}$ ref real-time wind direction comparison chart.

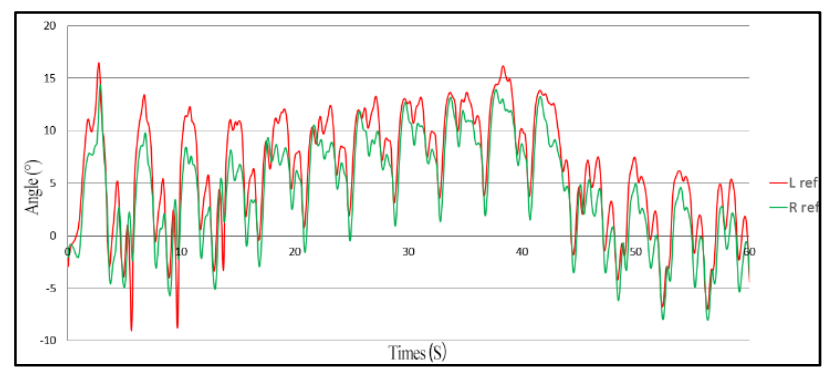

Fig. 14. vent $-8{ }^{\circ} \mathrm{L}$ ref, $\mathrm{R}$ ref real-time wind direction comparison chart.

\subsection{Comparison and selection}

The data of all wind speed monitoring points is converted into the wind direction angle, and the wind direction fluctuation curve is better than the position of $\mathrm{L}$ ref, $\mathrm{R}$ ref by comparing the wind direction real time curve.

The following Fig 15-22 is a real-time curve of wind direction for all monitoring points in the optimized area.

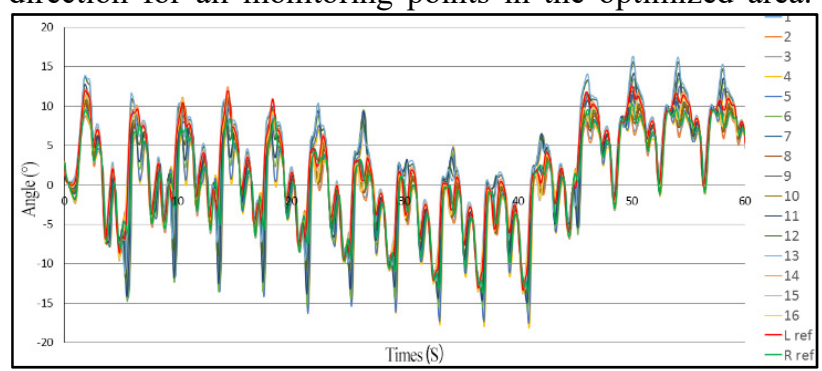

Fig. 15. Comparison of real-time wind direction of all monitoring points in the $8^{\circ}$ optimized area without ventilation

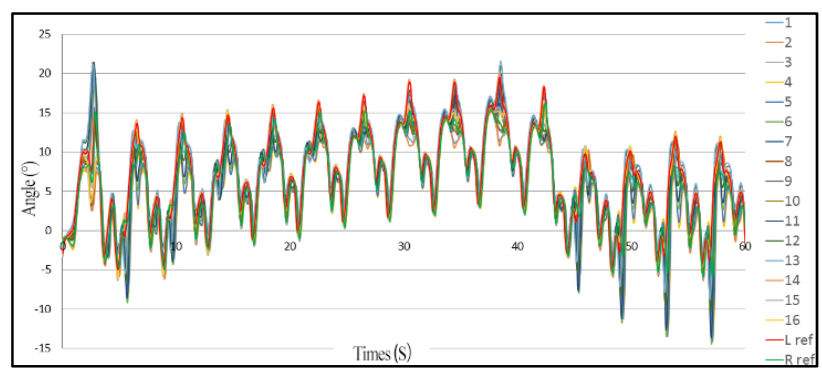

Fig. 16. Comparison of real-time wind direction of all monitoring points in the optimized area without venting $-8^{\circ}$

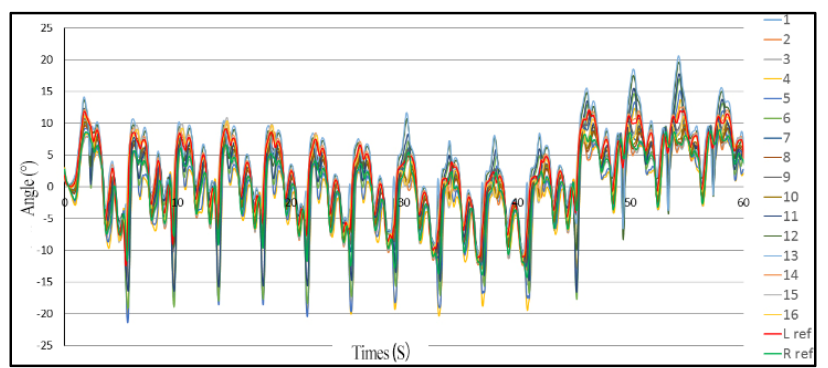

Fig.17. Comparison of real-time wind direction of all monitoring points in the $8^{\circ}$ optimization area with vents.

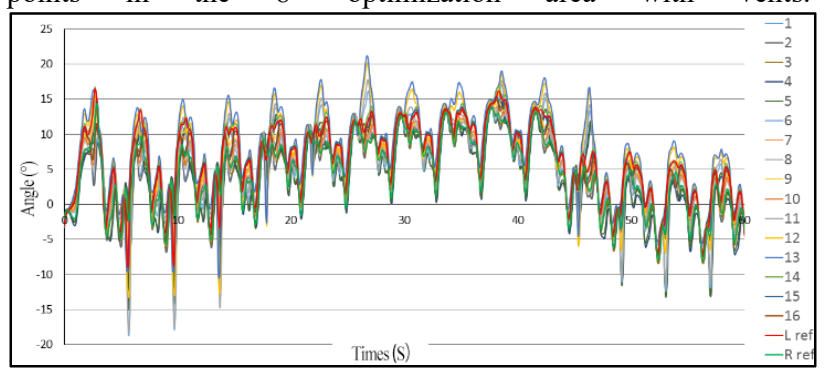

Fig. 18. Comparison of real-time wind direction of all monitoring points in the optimized area with vent $-8^{\circ}$.

The following is a comparison of the various sets of advantages with the real-time wind direction of $L$ ref, $R$ ref.

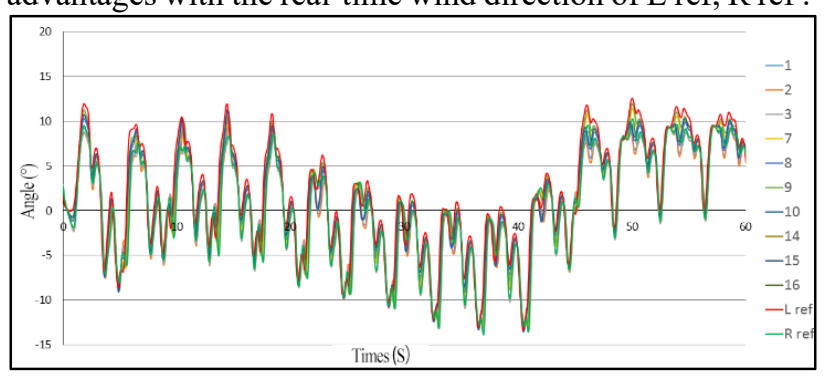

Fig. 19. Comparison of the advantages of $8^{\circ}$ without vents and $\mathrm{L}$ ref, $\mathrm{R}$ ref.

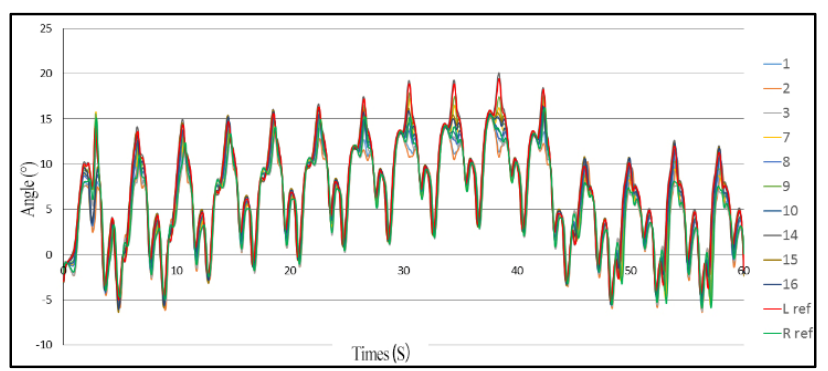

Fig.20. Comparison of the advantages of the vent-free $-8^{\circ}$ group and $\mathrm{L}$ ref, $\mathrm{R}$ ref. 


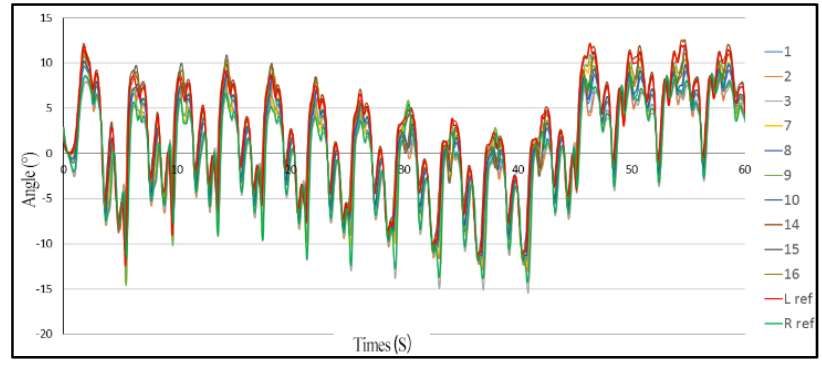

Fig.21. the advantages of $8^{\circ}$ vents and L ref, R ref.

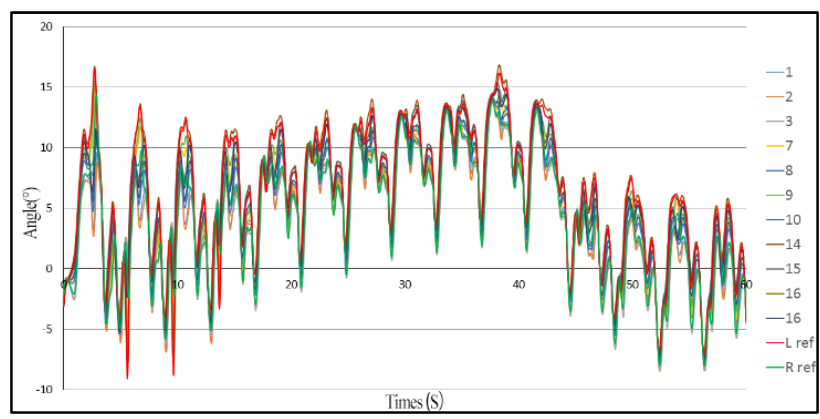

Fig.22. vent $-8^{\circ}$ group advantage and $\mathrm{L}$ ref, $\mathrm{R}$ ref comparison chart.

The following Fig 23-26 is the best advantage compared with the real-time wind direction of L ref, $\mathrm{R}$ ref.

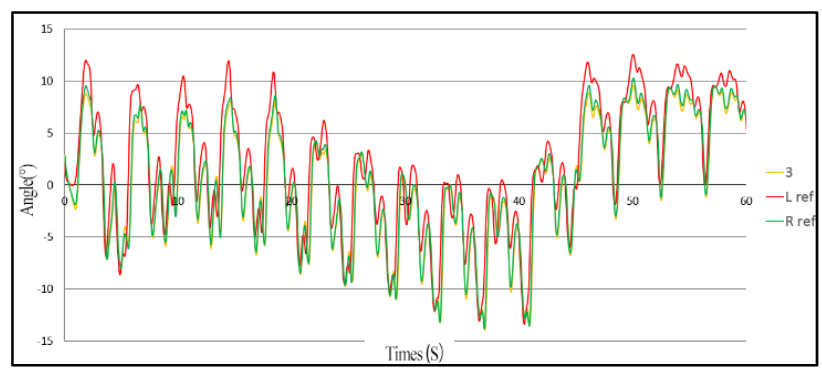

Fig.23. Comparison of the best advantage of $8^{\circ}$ without vents and $\mathrm{L}$ ref, $\mathrm{R}$ ref.

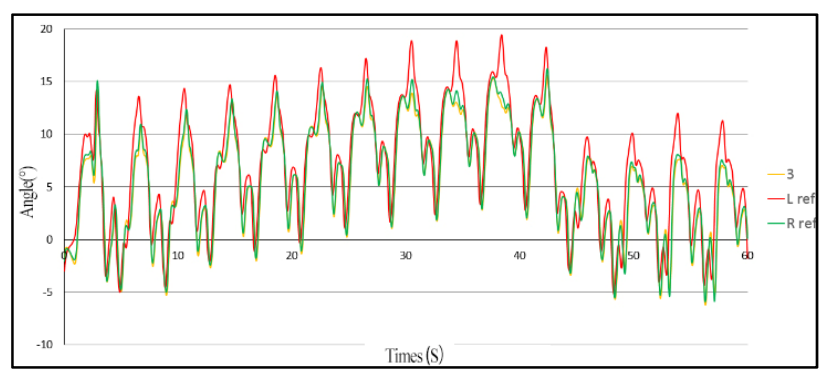

Fig. 24. No vent $-8^{\circ}$ best advantage and $L$ ref, R ref comparison chart.

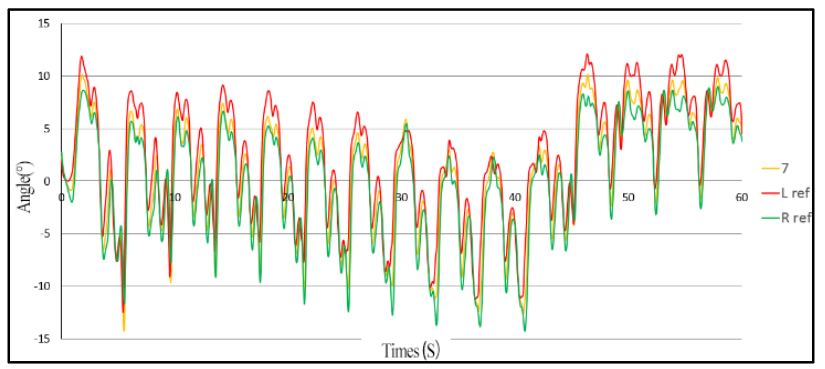

Fig. 25. the best advantage of $8^{\circ}$ vent and L ref, R ref.

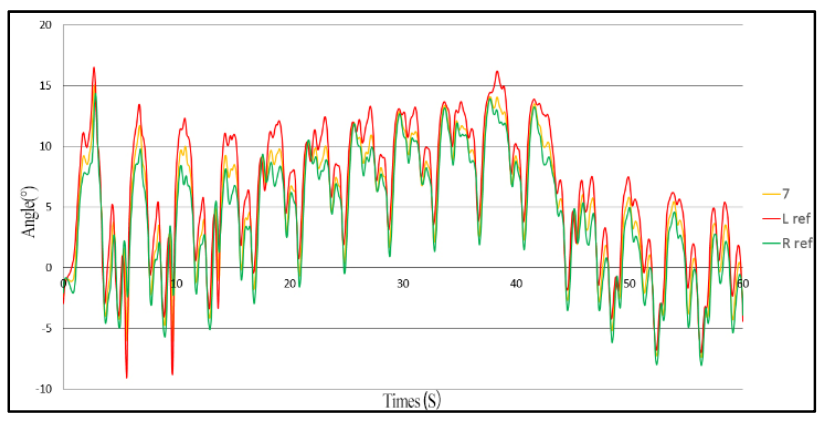

Fig. 26.the best advantage of vent $-8^{\circ}$ compared with $L$ ref, $R$ ref.

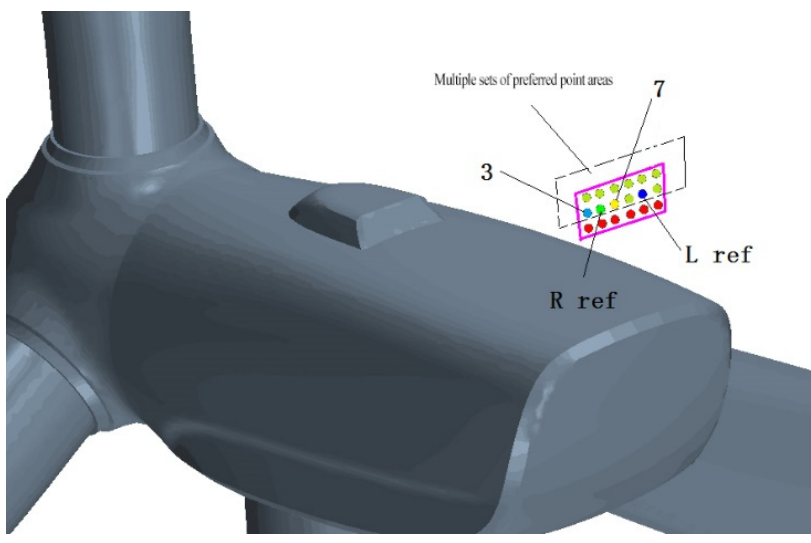

Fig. 27. The most advantageous relative position.

From the comparison results, the selected wind direction fluctuation is more stable than L ref, R ref, and the swing amplitude is smaller. It can also be seen that the flow direction of the wind is $8^{\circ}$ or $-8^{\circ}$, and the advantages of the multiple groups and the position of the most advantageous are unchanged, indicating that the change of the flow direction does not affect the distribution of the advantages and disadvantages of the wind direction fluctuation. The most advantageous coordinates are shown in Table 3 below, and the relative positions are shown in Fig. 27.

Table 3. Most advantageous coordinates

\begin{tabular}{|c|c|c|}
\hline case & $\begin{array}{c}\text { The most } \\
\text { advantageous } \\
\text { coordinates }\end{array}$ & coordinates $(\mathrm{x}, \mathrm{y}, \mathrm{z})$ \\
\hline
\end{tabular}




\begin{tabular}{|c|c|c|}
\hline $\begin{array}{c}\text { Without } \\
\text { vent }\end{array}$ & 3 Point & $\begin{array}{c}{[5.512131,-} \\
0.4274023578720121,- \\
0.3072180233953805] \mathrm{m}\end{array}$ \\
\hline With & 7 Point & $0.036843292016179086,-$ \\
vents & & $0.3194350141819121] \mathrm{m}$ \\
\hline
\end{tabular}

\subsection{Influence of wind direction on wind direction near reference point}

Compared the calculation results of the flow direction of $8^{\circ}$ and $-8^{\circ}$ with no ventilation, the influence of the wind direction on the wind direction near the reference point is investigated. The following Fig 28-30 are the real advantages of $\mathrm{L}$ ref, $\mathrm{R}$ ref and wind direction fluctuations in real-time comparison of wind direction with $8^{\circ}$ and $-11^{\circ}$ respectively.

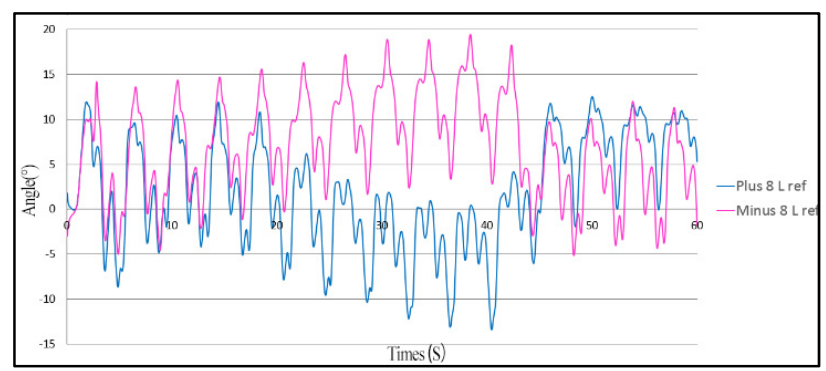

Fig. 28. Comparison of $8^{\circ}$ and $-8^{\circ} \mathrm{L}$ ref real-time wind direction without vents.

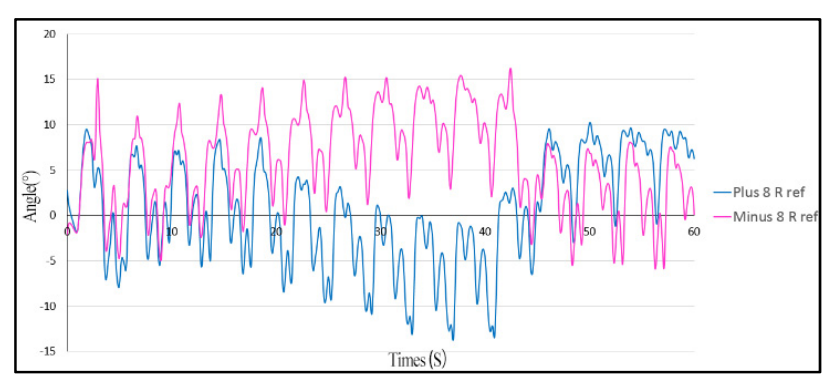

Fig.29. Comparison of real-time wind direction without vent $8^{\circ}$ and $-8^{\circ} \mathrm{R}$ ref.

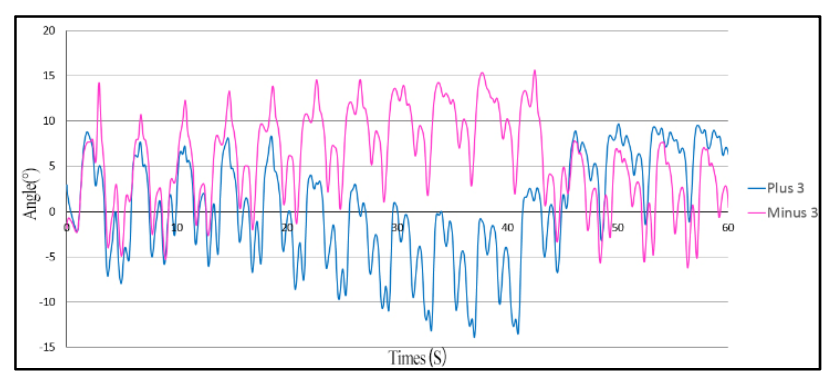

Fig. 30. Comparison of real-time wind direction without vent $8^{\circ}$ and $-8^{\circ}$.

From the comparison of real-time wind direction results, with the change of incoming wind direction, the wind direction near the reference point fluctuates in a single cycle, and the amplitude changes little, and the only change is the fluctuation trend of large-cycle oscillation. In the opposite direction of flow, the fluctuation trend of large-cycle oscillations is also reversed.

\subsection{Effect of venting on wind direction near reference point}

By comparing the calculation results of the vents in the $8^{\circ}$ state, the influence of the upper part of the nacelle plus the vents on the wind direction is investigated. The following contents are the best advantages of $\mathrm{L}$ ref, $\mathrm{R}$ ref and wind direction fluctuations in real-time comparison of the wind direction with or without vents in the $8^{\circ}$ state of the incoming wind direction.

From the comparison of real-time wind direction results, the wind direction fluctuations in the two states with or without vents are basically the same, and the addition of vents has little effect on the wind direction near the reference point.

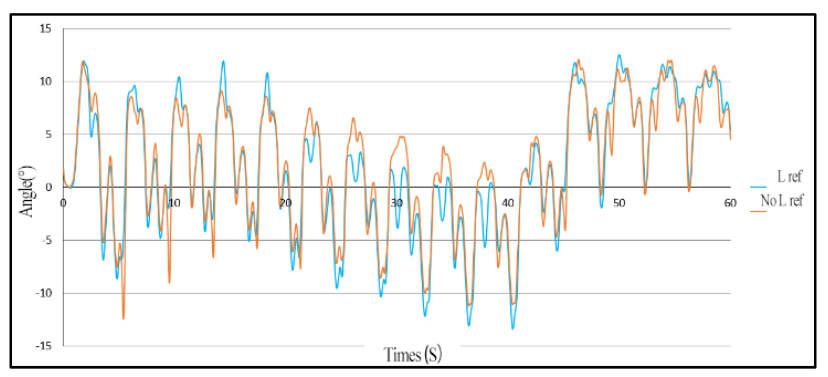

Fig. 31. Real-time wind direction comparison of $\mathrm{L}$ ref with or without vents.

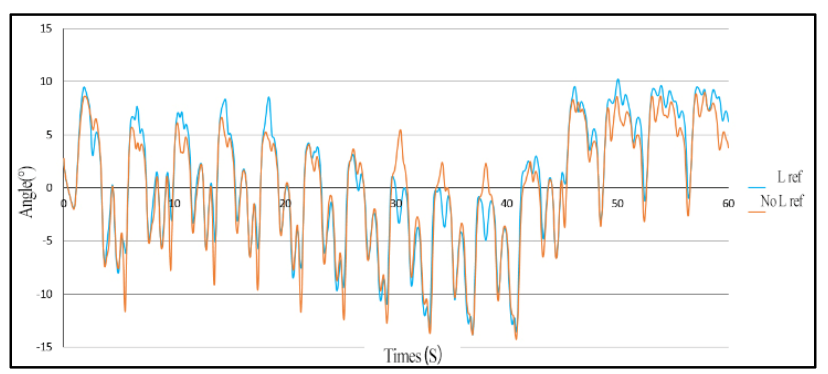

Fig. 32. Real-time wind direction comparison of $\mathrm{R}$ ref with or without vents. 


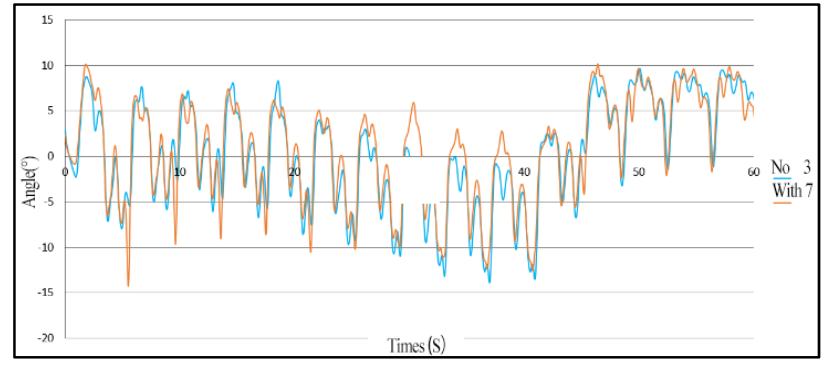

Fig.33. The best advantage of wind direction fluctuations is the real-time wind direction contrast with or without vents.

\section{Conclusions}

1. From the transient calculation results (speed, vortex dynamic map), it can be seen that the vicinity of the reference point is the region where the airflow is relatively intense. It is necessary to conduct in-depth CFD research on this flow phenomenon.

2. By comparing the real-time wind direction curve, the best advantage of wind direction fluctuation can be obtained, and the selected point wind direction fluctuation is more stable and the swing amplitude is smaller.

3. The change of the flow direction does not affect the distribution of the advantages and disadvantages of the wind direction fluctuation. In the case of incoming wind directions of $8^{\circ}$ and $-8^{\circ}$, the advantages of the multiple sets and the most advantageous positions remain unchanged and tend to be away from the surface of the nacelle.

4. With the change of the incoming flow direction, the fluctuation of the wind direction near the reference point in a single cycle (vibration shape, amplitude) does not change much, and the change is only the fluctuation trend in the large period of the oscillation, and the flow direction is opposite. The fluctuation trend in the big cycle of the shock is also reversed.

5. The wind direction fluctuations in the two states with or without vents are basically the same, and the addition of vents has little effect on the wind direction near the reference point.

The above results are obtained based on the analysis of the two wind direction conditions of the same wind speed. It is possible to calculate more wind speeds and different wind down characteristics for verification, and then make a conclusion after global comparison and optimization.

\section{References}

1. U. Datta, J. Shi, A. Kalam. Primary frequency control of a microgrid with integrated dynamic sectional droop and fuzzy based pitch angle control. Int. J. Electr. Power Energy Syst, 111, 248-259 (2019).

2. Z. Hameed, Y.S. Hong, Y.M. Cho, S.H. Ahn, C.K. Song. Condition monitoring and fault detection of wind turbines and related algorithms: A review. Renewable \& Sustainable Energy Reviews, 13, 1-39 (2009).

3. R. Hemmati. Optimal design and operation of energy storage systems and generators in the network installed with wind turbines considering practical characteristics of storage units as design variable. Journal of Cleaner Production, 185, 680-693 (2018).

4. H. Li, Z. Chen. Overview of different wind generator systems and their comparisons. Iet Renewable Power Generation, 2, 123-138 (2008).

5. Z.X. Li, Y. Jiang, Q. Guo, C. Hu, Z.X. Peng. Multidimensional variational mode decomposition for bearing-crack detection in wind turbines with large driving-speed variations. Renew. Energy, 116, 55-73 (2018).

6. M. Liserre, R. Teodorescu, F. Blaabjerg. Stability of photovoltaic and wind turbine grid-connected inverters for a large set of grid impedance values. Ieee Transactions on Power Electronics, 21, 263-272 (2006).

7. J. Morren, S.W.H. de Haan, W.L. Kling, J.A. Ferreira. Wind turbines emulating inertia and supporting primary frequency control. Ieee Transactions on Power Systems, 21, 433-434 (2006).

8. L.J. Vermeer, J.N. Sorensen, A. Crespo. Wind turbine wake aerodynamics. Progress in Aerospace Sciences, 39, 467-510 (2003).

9. P. Bechtle, M. Schelbergen, R. Schmehl, U. Zillmann, S. Watson. Airborne wind energy resource analysis. Renew. Energy, 141, 1103-1116 (2019).

10. U. Bhardwaj, A.P. Teixeira, C.G. Soares. Reliability prediction of an offshore wind turbine gearbox. Renew. Energy, 141, 693-706 (2019).

11. X.F. Wang, X.W. Zeng, J.L. Li, X. Yang, H.J. Wang. A review on recent advancements of substructures for offshore wind turbines. Energy Conversion and Management, 158, 103-119 (2018).

12. B. Yang, T. Yu, H.C. Shu, J. Dong, L. Jiang, Robust sliding-mode control of Wind energy conversion systems for optimal power extraction via nonlinear perturbation observers, Applied Energy, 2018, pp. 711723. 